\title{
Essais
}

Revue interdisciplinaire d'Humanités

Narration et lien social

\section{Récit, Architecture, Milieux}

\section{Marc-Antoine Durand}

\section{(2) OpenEdition}

Journals

Édition électronique

URL : https://journals.openedition.org/essais/9229

DOI : 10.4000/essais. 9229

ISSN : 2276-0970

\section{Éditeur}

École doctorale Montaigne Humanités

\section{Édition imprimée}

Date de publication : 15 mai 2013

Pagination : 53-77

ISBN : 978-2-9544269-0-7

ISSN : 2417-4211

\section{Référence électronique}

Marc-Antoine Durand, «Récit, Architecture, Milieux », Essais [En ligne], 3 | 2013, mis en ligne le 01 septembre 2021, consulté le 09 septembre 2021. URL : http://journals.openedition.org/essais/9229 ; DOI : https://doi.org/10.4000/essais.9229 


\section{Récit, Architecture, Milieux}

\section{Marc-Antoine Durand}

Le laboratoire GERPHAU (philosophie - architecture - urbain UMR 7218 MCC/CNRS - LAVUE) développe une interface privilégiée entre la recherche en sciences humaines et la maitrise d'œuvre urbaine et paysagère. Associant chercheurs et praticiens autour de la notion d'Architecture des Milieux, les deux recherches / actions présentées ici - l'aménagement du Secteur Sud-Ouest (SSO) de Rennes (étude urbaine dont la maîtrise d'œuvre fut confiée par Rennes Métropole à OBRAS - architectes, Paris) et l'aménagement du parc de Senne à Bruxelles (opération de maitrise d'œuvre confiée par l'IBGE - Institut Bruxellois pour la Gestion de l'Environnement - aux paysagistes de La Compagnie du Paysage, à Paris) - explorent, par l'expérimentation, le récit en tant qu'outil de représentation et de conception des territoires mis en transformation.

Comme le rappelle l'architecte Frédéric Bonnet (OBRAS), l'Architecture des Milieux est une hypothèse de travail fondée sur l'idée que l'architecture est l'art de "créer des liens, l'art de construire n'en étant qu'une modalité particulière $»^{1}$. Si l'on remet en cause le grand récit progressiste moderne, qui a confié "à l'avenir la fonction de rendre compte du sens de développement »", à la manière dont Paul Ricœur s'est exercé à le faire dans sa célèbre trilogie des Temps et Récit, il nous est possible d'entrevoir les possibilités du récit en tant qu'outil du liant (ou du reliant). Le philosophe, en publiant en 1998 un article intitulé Architecture et Narrativité, a ouvert la porte à un rapprochement analogique entre les fonctions liantes (ou reliantes) de l'architecture et du récit, la première comme opération configurante de l'espace, la seconde comme opération configurante du temps, le parallélisme de la démonstration allant même jusqu'à proposer une inversion des termes.

Frédéric Bonnet, "Architecture des Milieux », Le Portique, n² 25, 2010, p. 129-136.

2 Chris Younès, Stéphane Bonzani, «Représenter, imaginer, projeter : éléments de méthode pour une architecture des milieux ", Le Portique, $\mathrm{n}^{\circ} 25,2010$, p. 53-58. 
Cet entremêlement du temporel et du spatial qualifie aujourd'hui l'expérience habitante contemporaine, cet éco-habiter métropolitain qu'il a s'agit pour nous de décrire et de rendre actif par la mise en récit des territoires et des mémoires rencontrés.

Mêlant données quantitatives (rapports, études scientifiques) et données qualitatives (observations, descriptions, entretiens) la méthode que nous avons développée, la philosophe Chris Younès et moi-même, est mixte. Elle travaille à l'articulation des récits de vie au territoire qu'ils racontent, à la mémoire qu'ils portent. Elle vise à rendre compte des situations habitantes dans la géographie, pratiquée ou imaginaire, du site, et dans la boucle du temps (passé - présent futur) ; par le récit, en tant qu'il est la représentation des actions humaines dans l'espace et dans le temps : il raconte nos lieux de vies et nos ailleurs, il est porteur de souvenirs en même temps que d'attentes. En outre, ce sont les vertus emphatiques du récit que nous allons tenter ici de décrire.

\section{Récits de vie et territoires / Cas $\mathbf{n}^{\circ} 1$ : Rennes SSO}

\section{Territoire et limites habitées}

\subsection{Rennes Métropole : une culture des limites}

La métropole rennaise a depuis longtemps fait le choix de la "ville-archipel ». Cependant, ces dernières années, et plus encore aujourd'hui, à l'heure de la taxe carbone, ce modèle est mis à mal par l'allongement des temps de déplacement qu'il induit. La tentation alors est grande de réveiller le spectre d'une périurbanisation étirée sur les axes de connexions, comme ceux qui traversent le SSO, et surtout comme le développement du nouveau centre de SaintJacques-la-Lande semble le proposer. Le décentrement opéré par ce nouveau centre $^{3}$ pose en effet clairement la question de la forme urbaine à l'échelle métropolitaine comme centrale dans le projet d'aménagement du site proposé ici. Sur ce point nous nous positionnerons rapidement en faveur d'un retour à la ville-archipel, en ce qu'elle induit, du point de vue des modes de vie de ses habitants et usagers, bien plus d'avantages que de contraintes, comme nous allons le voir.

3 Voir à ce sujet le livre de Laurent Devisme, La ville décentrée : figures centrales à l'épreuve des dynamiques urbaines, l'Harmattan, 2005. 


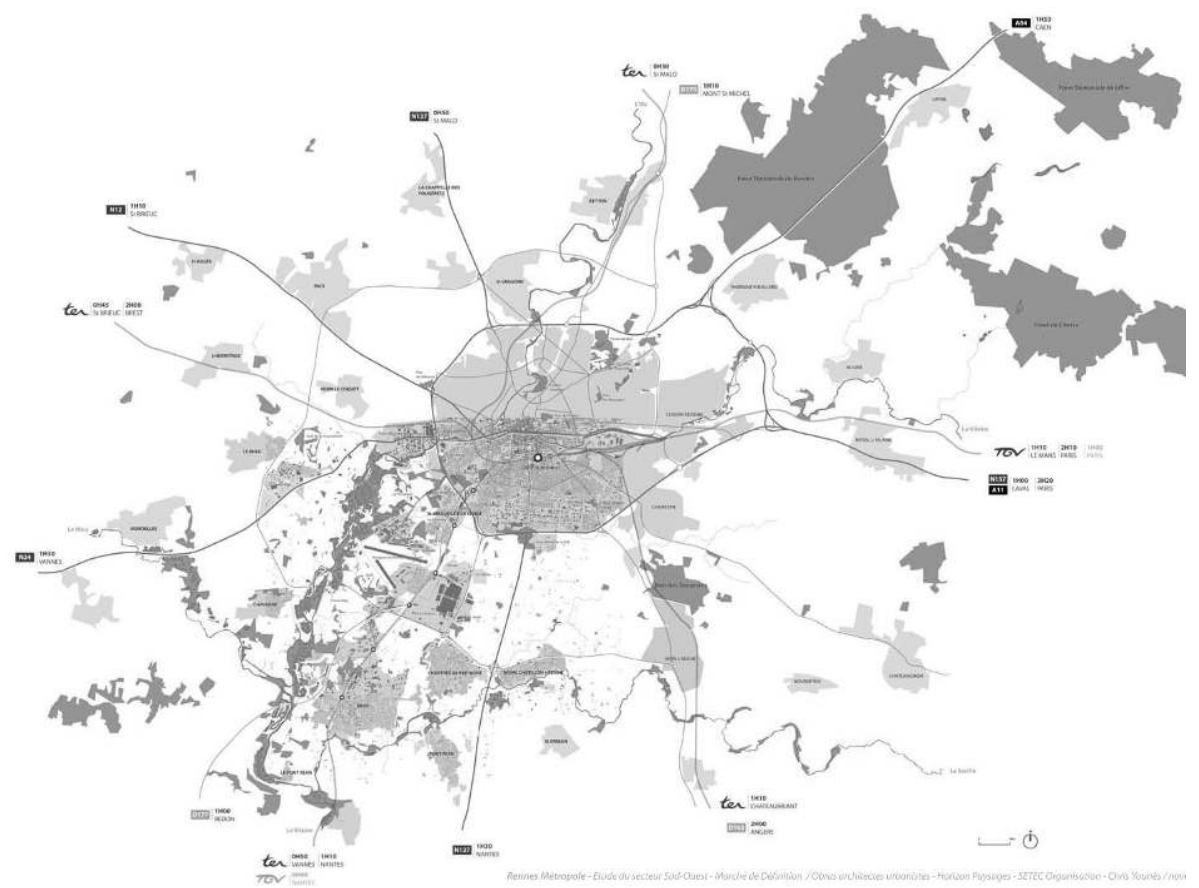

Illustration 1. Rennes : ville archipel, carte de la métropole rennaise (source OBRAS).

Selon nous, ce retour ne peut se faire sans une actualisation du concept de limite. C'est en effet la qualité des limites qu'elle met en œuvre qui font les vertus de la ville-archipel. Leur netteté est facteur de qualité de vie : le resserrement du cadre bâti en entités spatiales clairement définies facilite l'identification et l'appropriation des territoires, la multiplication et la distinction des îlots permet l'individuation, facilite l'orientation, la densité de ces îlots favorise le développement de réseaux de proximités locaux... En outre, l'interaction ville / nature y est d'autant plus forte que les limites sont franches.

Par ailleurs, cette culture des limites de Rennes Métropole ancrée dans l'histoire de son développement en fait sa singularité, et comme le souligne Jean-Yves Chapuis c'est un sérieux atout ${ }^{4}$. L'expérience le vérifie facilement, un tour de rocade par exemple suffit pour constater la qualité de traitement des bords dans la métropole rennaise, sur certaines portions l'infrastructure routière délimite strictement la ville des champs, c'est un cas unique en France.

Dans un rapport homothétique au développement métropolitain, le site en lui-même présente un développement en îlots ou en tâche. Il porte en lui cette culture des limites. Et de même que la "tentation d'étirement" se fait sentir à l'échelle métropolitaine, elle est présente localement, dans les extensions

4 Jean-Yves Chapuis, « De la ville historique à la ville archipel », Études Foncières, n 105, ADEF, septembre-octobre 2003. 
pavillonnaires du centre de Bruz, ou à Noyal-Chatillon-sur-Seiche par exemple. Ainsi, les questionnements locaux et métropolitains s'emboîtent.

Historiquement déjà, le SSO pourrait être raconté par l'histoire de ses limites. Le souci de la bonne limite s'est en effet posé à deux reprises dans l'histoire récente : au milieu du XIX ${ }^{e}$ siècle lorsqu'a été déplacée la frontière Bruz-Chartes-de-Bretagne ${ }^{5}$, puis tout récemment en 1992 lors de la fusion Noyal-Chatillon-sur-Seiche. Ces dernières années le recentrement de la commune de Saint-Jacques-de-la-Lande a provoqué un déplacement puis un brouillage des limites non pas administratives mais physiques, ce qui a initié comme nous le disions plus haut un phénomène de dillution urbaine qui pose question aujourd'hui.

Les limites naturelles que constituent la Seiche et surtout la Vilaine ont contribué au développement du territoire de deux manières bien distinctes : si l'on peut dire de la vallée de la Seiche qu'elle est habitée (les extensions pavillonnaires des lotissements de Noyal-Chatillon-sur-Seiche sont pour ainsi dire à " flanc de vallée "), celle de la Vilaine, rendue difficile d'accès par les carrières de sable qui l'ont élargie, est finalement très peu aménagée au regard du potentiel paysager qu'elle représente.

Les grandes infrastructures métropolitaine que sont les axes routiers Rennes-Redon et Rennes-Nantes, qui participent du modèle de la ville-archipel en reliant les satellites à la ville centre, sont des coupures importantes dans le territoire, tout comme l'aéroport Rennes-St-Jacques. Cependant leur potentiel paysager suscite l'intérêt ${ }^{6}$, ce sont aujourd'hui les entrées principales du territoire, c'est par elles qu'il se donne à voir.

\subsection{Habiter les limites contemporaines}

La condition urbaine contemporaine met à mal l'idée même de limite, elle la fait tendre vers ses deux extrémités que sont l'absence de limite et la limite finie, l'illimité et le ségrégant. La ville diffuse, sans limite, qui s'étire à perte de vue, et la gated community seraient ainsi les figures extrêmes du concept limite. Le SSO, au contraire, nous semble décliner une diversité de limites des plus intéressantes et offrir des conditions d'habitabilité riches et variées. C'est ce que nous nous proposons d'étudier plus en détail.

Pour interroger ces expériences habitantes des limites nous avons choisi sur le site quelques entités spatiales représentatives d'une figure de limite.

5 Document : Changement de limite entre Chartes et Bruz, imprimerie de A. Marteville, Archives départementales d'Ille-et-Vilaine, 1852.

6 Voir notamment l'article de Régis Guignard, "La Nationale 137 : un jardin extraordinaire ", Place Publique, $\mathrm{n}^{\circ} 17$, septembre 2009. 
- La Courrouze : la pièce manquante

Le nouveau quartier de la Courrouze est situé en bordure de rocade à Rennes sur une zone de friches militaro-industrielles. Le pari du projet est d'en faire le lien manquant entre le centre de Rennes et la vallée de la Vilaine. L'expérience habitante violente qu'aurait pu être celle d'habiter à proximité de la rocade se change en expérience éco-habitante exemplaire.

- La vallée de la Vilaine : la limite épaisse

La vallée de la Vilaine représente la rupture la plus forte du territoire SSO. Si depuis Rennes un travail de connections est amorcé c'est encore une limite épaisse trop faiblement poreuse et quasiment inhabitable dans le sens transversal.

- La Morinais : la limite devient floue

Le nouveau centre St-Jacques-de-la-Lande (la Morinais) est à l'origine d'un déplacement, et d'un brouillage de la limite centre / périphérie selon l'axe Rennes Redon.

- L’aéroport : la limite de Tantale

L'aéroport Rennes-St-Jacques est une plaque posée au milieu du territoire SSO, une zone aux limites infranchissables mais invisibles, qui illustre d'une certaine manière le supplice de Tantale : la vallée de la Vilaine est visible, juste là, mais tout à fait inaccessible, intouchable.

- PSA (site la Janais) : la masse limite

Le site PSA (la Janais) représente une « zone interdite » d'une dimension comparable à celle de l'aéroport qui constitue, comme celle-ci, une rupture importante du continuum spatial du territoire SSO, mais à la différence de l'aéroport, le site PSA impacte considérablement le paysage.

- Le quartier St-Jacques aéroport : l'étau

Le quartier St-Jacques aéroport constitue les restes de l'ancien centre de Saint-Jacques-de-la-Lande. Sa situation particulière, « en étau » entre l'aéroport et le site de la Janais, en fait une entité spatiale singulière intéressante du point de vue d'une expérience habitante des limites.

- Le centre de Bruz : la limite se délite

L'extension pavillonnaire centre sud de Bruz fausse le développement concentrique initial de la commune. Sa limite se délite.

- Le campus de Ker Lann : la limite poreuse

Les constructions implantées sur le campus de Ker Lann prennent place dans un environnement verdoyant particulièrement soigné. Leur faible densité travaille un rapport poreux à l'extérieur et à l'environnement immédiat.

- Le golf de Cicé-Blossac : l'enclos

Le golf de Cicé-Blossac est un lotissement clos de maisons individuelles entre le centre de Bruz et la Vilaine. La limite symbolique de cette spatialisation de «l'entre soi » est forte. 
L'expérience de la limite est toujours celle de son dépassement, de son franchissement. C'est toujours l'expérience d'un passage, d'un mouvement, d'un lien; et ce bref état des lieux de la question sur le territoire SSO témoigne de la diversité et de la richesse potentielle de ces expériences sur le site. Comme le remarque le philosophe Jacques Derrida l'expérience de la limite est en fait celle de la limitrophie (trophie : qui se nourrit au contact de), celle de l'espace limitrophe, c'est-à-dire celle de l'espace que génère la limite. L'expérience d'un " entre-espace " de la limite. On voit ainsi comment la limite travaille à l'articulation et au ménagement des territoires et peut permettre une gestion pacifiée des proximités spatiales immédiates, ce qui est essentielle.
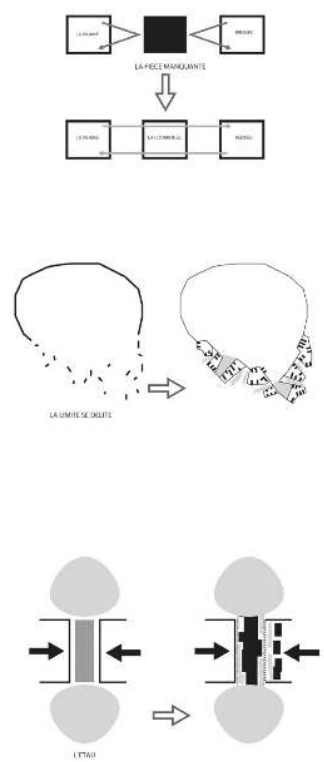
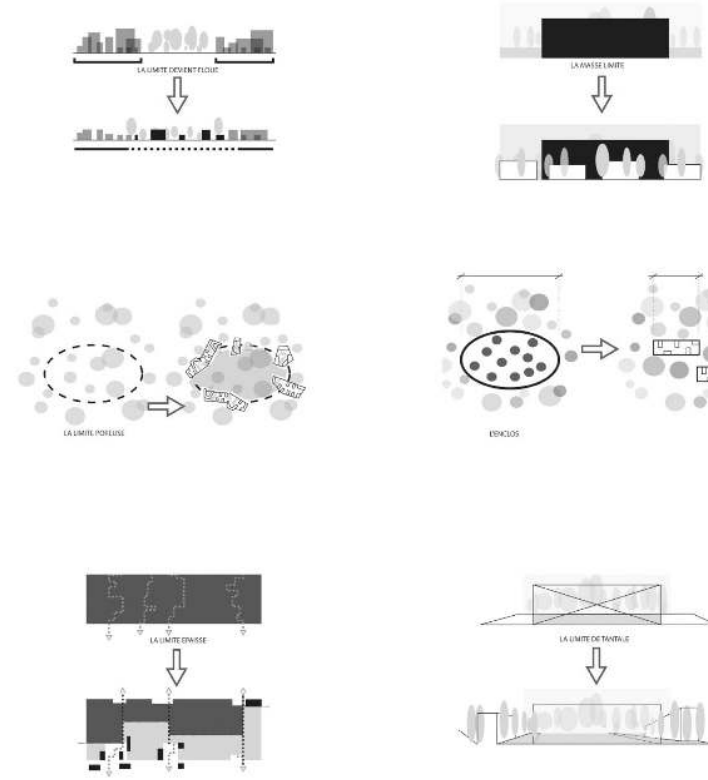
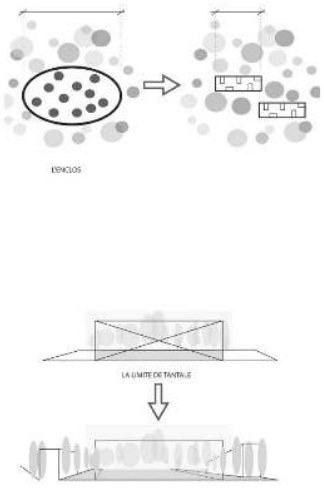

Illustration 2. Du concept à l'action : la boîte à outil, schémas de principe représentant une déclinaison des différentes expériences habitantes de la limite (source : MAD + OBRAS. De haut en bas et de gauche à droite : la pièce manquante, la limite floue, la masse limite, la limite se délite, la limite poreuse, l'enclos, l'étau, la limite épaisse, la limite de Tantale).

Aujourd'hui cependant la condition urbaine contemporaine met à mal ce concept, les logiques de juxtaposition sont devenues insuffisantes pour penser un monde devenu global, incertain et inquiet, pour penser un monde où l'équilibre des cohabitations entre les milieux (humains et naturels) est plus que jamais remis en question. Aussi il semble nécessaire de penser de nouvelles relations actives, complémentaires, symbiotiques afin de susciter de nouvelles conditions vivifiantes de ménagement, d'aménité, de solidarité. Cette pensée de la relation active est celle qui aujourd'hui vient actualiser le concept de limite tel que nous l'avons exposé jusqu’à présent. Cette pensée est celle de la Reliance.

7 Chris Younes, Vers une architecture des milieux comme art de la reliance, publication Europan et 


\title{
2. Territoire et reliances habitantes
}

\subsection{Rennes SSO : l'enjeu des reliances}

\begin{abstract}
"Notre civilisation sépare plus qu'elle ne relie. Nous sommes en manque de reliance, et celle-ci est devenue besoin vital ; elle n'est pas seulement complémentaire à l'individualisme, elle est aussi la réponse aux inquiétudes, incertitudes et angoisses de la vie individuelle. Parce que nous devons assumer l'incertitude et l'inquiétude, parce qu'il existe beaucoup de sources d'angoisse, nous avons besoin de forces qui nous tiennent et nous relient. Nous avons besoin de reliance parce que nous sommes dans l'aventure inconnue. »
\end{abstract}

Edgar Morin

Le concept de Reliance est inventé par le sociologue Marcel Bolle de Bal dans le but premier de dépasser celui de Relation, qu'il jugeait épuisé. D’un point de vue social il définit ainsi la Reliance " comme le partage des solitudes acceptées et l'échange des différences respectées $» .{ }^{8}$ Mais l'intérêt du concept tient aussi, et surtout, dans le fait qu'il qualifie la "relation » ou le «lien " dans ce qu'il active chez les acteurs concernés. C'est ce point particulièrement qui intéressera Edgar Morin, à qui l'on doit une généralisation épistémologique du concept de Reliance au travers sa méthode dite Complexe : " La notion de reliance, inventée par le sociologue Marcel Bolle de Bal, comble un vide conceptuel en donnant une nature substantive à ce qui n'était conçu qu'adjectivement, et en donnant un caractère actif à ce substantif. "Relié" est passif, "reliant" est participant, "reliance" est activant $»$.

Pour Morin, la reliance est « l'action de relier et de se relier et ses résultats ", elle dit la relation de A à B autant que les effets de la relation sur $\mathrm{A}$ et sur $B$, elle quantifie le lien en même temps qu'elle le qualifie. La Reliance, pour Morin, c'est le fondement épistémique, mais aussi pragmatique et éthique d'une " écologie de l'action" nécessaire à tous et à chacun.

La Reliance est une action, c'est aussi pour Edgar Morin une méthode qui condense les principes de la pensée Complexe, et parmi ceux-ci la dialogique : qui cherche à unir ce qui se contredit pour exprimer une réalité " complexe ". "La dialogique postule et tient pour identifiable et par là intelligible bien qu'inexplicable in fine, la conjonction du complémentaire et de l'antagoniste, du pour et du contre, "d'homo sapiens et d'homo démens", de l'homogénéisant et de l'hétérogénéisant, de l'ordre et du désordre, du continu et du discret, de l'unité et de la pluralité ; "toutes choses étant causées et causantes,

ville durable, septembre 2009 (concours européen d'architecture et d'urbanisme durable).

8 Jean-Louis Le Moigne, Edgar Morin, «Le génie de la Reliance », Synergies Monde, n 4, 2008, p. 178.

9 Edgar Morin, La méthode, tome 4 : L'éthique, Éditions du Seuil, 2004. 
médiates et de l'immédiate..." " ${ }^{10}$ Ce principe dialogique confère à la Reliance et à cette " écologie de l'action » dont Edgar Morin se fait le porte-parole, une puissance heuristique qui permettrait de contrer "les effets mutilants, "décivilisants", de la séparation de la culture du citoyen, qui fait (ou qui "applique", présumé sans chercher à comprendre), et de la culture des experts qui sont présumés comprendre sans avoir à faire. ${ }^{11}$ C'est là tout ce qui nous intéresse dans le cadre d'un projet d'aménagement de territoire, répondre à cette nécessité que l'on a à travailler au partage des cultures habitantes et savantes. Si le dialogisme, tel que l'entendait Mikhail Bakthine ${ }^{12}$, est l'interaction qui se constitue entre le discours propre de l'énonciateur et les discours qui lui sont extérieurs. Nous nous intéresserons quand à nous à l'interaction qui se constitue (ou qu'il reste à constituer) entre les différents discours habitants et leurs pendants savants. L'usage du récit est à ce titre précieux.

Appliquer le concept de Reliance aux milieux habités c'est travailler les liens de soi (individuel ou collectif) aux milieux naturels, nous parlerons alors de Reliance Nature / Culture, ou de soi aux autres, et nous parlerons de Reliance Culture / Culture. Les reliances Nature / Culture activent des dispositifs d'alliances et de cohabitation entre les établissements humains et les milieux naturels. Elles relient l'habitant aux éléments (eau, air, terre), au vivant (faune et flore), au paysage, au dehors. Les reliances Culture / Culture activent quant à elles des solidarités sociales, des partages politiques du territoire. Elles relient l'habitant à l'Autre en travaillant des rapports sociaux-culturels : ruraux / urbains, classes aisées / classes modestes, habitants / migrants... des rapports d'échelle : local / métropolitain / national / global / mondial, centre / périphérie... des rapports poétiques : réel / imaginaire...

La Reliance est un concept spatial et temporel, c'est l'acte de relier dans l'espace et dans le temps. Chaque dispositif de Reliance réinsère ce qu'il relie dans la boucle du temps. En faire l'expérience c'est toujours se replacer à la fois dans un rapport de soi à l'héritage, à la mémoire et dans un rapport de soi à l'avenir. Les reliances activent les souvenirs et les aspirations.

Notre hypothèse est de dire qu'à l'heure du durable l'expérience habitante de la Reliance est aujourd'hui celle de l'éco-habiter métropolitain. Une expérience vécue comme aspiration collective partagée de cohabitation pacifiée à la fois avec ses semblables et avec le milieu naturel environnant. Comme nous allons le montrer, le territoire du SSO véhicule déjà, dans une certaine mesure, cette image d'un futur apaisé où la proximité avec la nature s'allie à la mixité

10 Jean-Louis Le Moigne, op. cit.

11 Idem.

12 Mikhail Backthine est un historien et théoricien russe de littérature, mort en 1975 il fut un précurseur de ce que l'on appelle la sociolinguistique. Il est cité par Ricœur dans l'article Architecture et Narrativité à propos de son concept de Chronotope. 
sociale pour améliorer la qualité de vie de ses habitants. Dans l'ensemble les dispositifs de reliance observés vont dans ce sens, et c'est ce que nous souhaiterions encourager. Nos paramètres d'appréciation des dispositifs de reliance sont ainsi fonction de cette aspiration à l'éco-habiter métropolitain.

\subsection{Rennes SSO : récits}

Les études consultées et les rencontres faites sur le terrain dessinent le visage d'un territoire riche en expériences habitantes. Afin de rendre compte des dispositifs de reliances et de déliances en acte sur le site nous avons choisi la forme du récit. Ces récits sont chaque fois basés sur des données objectives (les études) et réelles ou prises sur place (rencontres, discussions, impressions), ils ont tous été recomposés fictivement. Ce sont en quelque sorte des récits d'hypothèses fondées.

Nous avons choisi huit récits, qui chacun mettent en scène des habitants ou usagers du territoire SSO dans des situations habitantes significatives de la diversité des modes de vies rencontrés. Le choix du récit met l'accent sur l'affect qui travaille chacun de nous, il nous apparait comme le mode de représentation le plus en phase avec l'étude des dispositifs de reliance, qui active les liens et les mouvements affectifs entre les humains et le lieu de leur établissement : ce qui les pousse vers ce qu'ils aiment, ce qui leur fait fuir ce dont ils ont peur, ce qui les fait aller vers ce à quoi ils aspirent. 
2.2.1. Un endroit pour vivre / A. et C., jeune coupleà la Courrouze

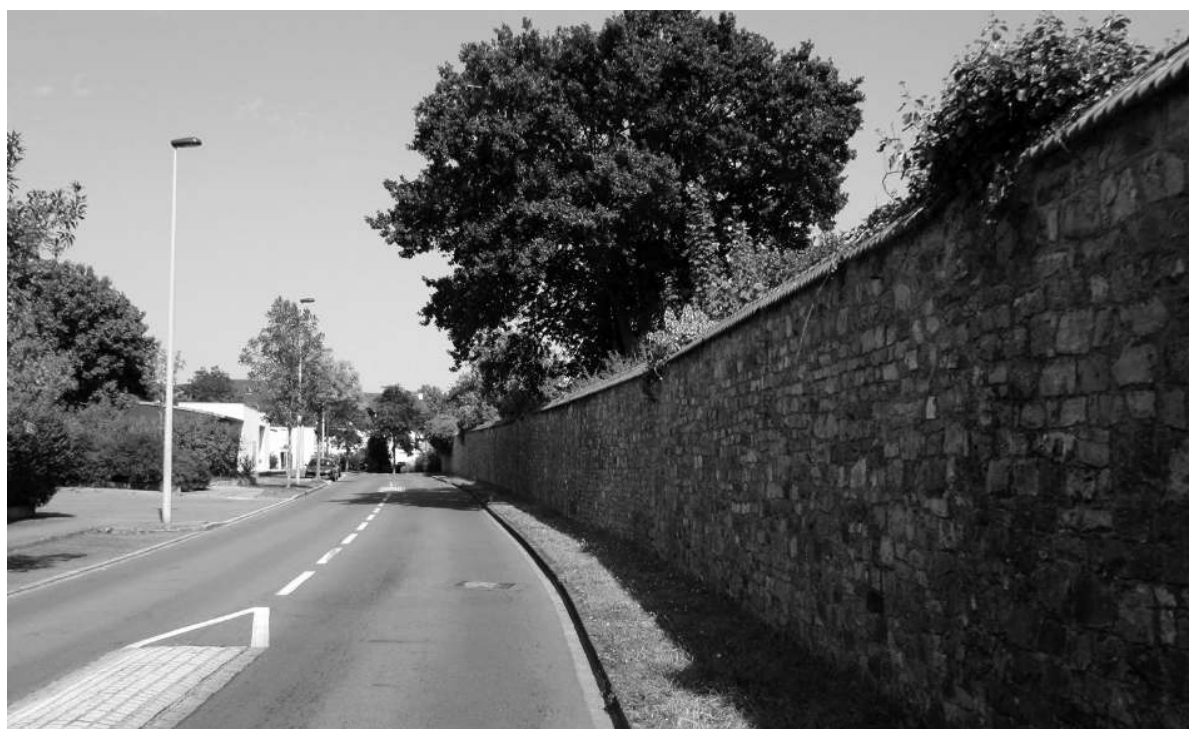

Illustration 3. Un ancien mur d'enceinte militaire conservé (source : MAD).

A. et C. vont emménager à la Courrouze et savent expliquer leur choix. Ils souhaitaient vivre en ville, c'est-à-dire au contact des autres, à proximité des commerces et de leur lieu de travail, ce qu'ils ont trouvé ici, dans un quartier qui sera dense et mixte (+ reliance : mixité sociale). Il ne voulait pas vivre en périphérie : de leur futur appartement le centre ancien de Rennes sera accessible (+ reliance : accès au centre) à pied par la coulée verte qui traverse le quartier, ou par les transports en commun.

Le côté clos du projet les inquiétaient cependant, son côté caché ; mais la mixité des programmes (équipements, services, logements...) et la diversité des connections aux réseaux de déplacements (+ reliance : accès aux mobilités) (doux ou motorisés) les ont vite rassuré : le quartier sera vivant, ce ne sera certainement pas une enclave!

Ils souhaitaient vivre au contact de la nature (+ reliance : proximité de la nature), et là encore, le quartier répond à leurs attentes : ils habiteront dans un parc, la Prévalaye et la vallée de la Vilaine seront à quelques minutes à pied, des jardins verront bientôt le jour.

Ils se refusaient aux quartiers neufs, à ces boîtes de bétons qu'ils voyaient pousser de-ci de-là, niant le contexte environnant, niant l'histoire des lieux, et les voilà dans un site occupé depuis plus d'un siècle par des activités militaires et industrielles. La présence de vestiges (+ reliance : héritage du passé, ancrage) les aura complétement convaincu : le grand mur qui longe le boulevard Mermoz et le tracé visible de l'ancienne voie ferrée exprimant le passé du lieu. 
A. et C. pensent avoir trouvé l'endroit idéal pour fonder une famille, ils pensent avoir trouvé le lieu qui répond à ce qu'ils attendent du futur $(++$ reliance : projection dans le futur).

2.2.2. La ville à la campagne, c'est tout de même la ville / M., retraité à la Morinais

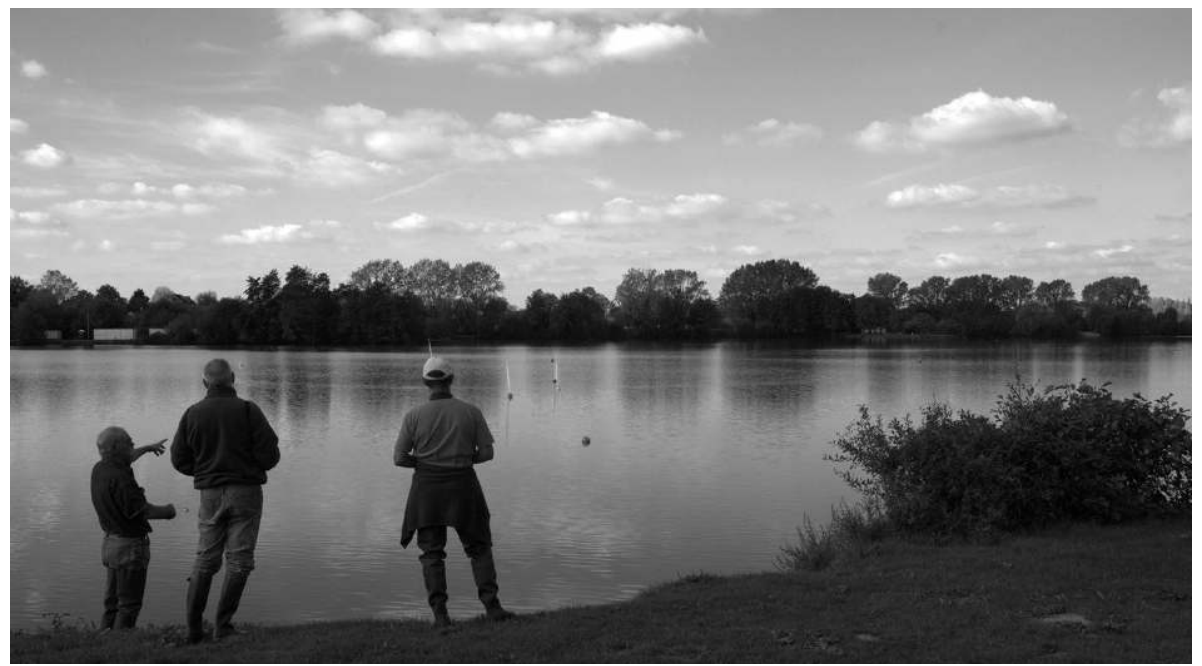

Illustration 4. Frégate de modèles réduits sur les étangs d'Apigné (source : MAD).

M. a longtemps pensé que son idéal résidentiel se situait à la campagne. Ce n'est qu'après le décès de sa femme qu'il s'est décidé à se rapprocher de ses enfants et du centre de Rennes. Ses aspirations et son budget lui ont fait choisir la Morinais, choix qu'il ne regrette en rien, même si la mixité sociale affichée du quartier ne le rassurait pas vraiment au départ. Il aurait aimé un endroit plus " tranquille ", mais a constaté que la coprésence de milieux sociaux différents (++ reliance : dans les années 1990, le maire de St-Jacques-de-la-Lande justifiait son projet : "La ville c'est aussi le frottement. Et le frottement c'est ce qui crée la chaleur.") et des générations n'induisait pas nécessairement la mixité relationnelle qu'il craignait.

La proximité de la nature (+ reliance : proximité nature) est pour lui essentielle, en même temps que les possibilités publiques de déplacement (+ reliance : accessibilité), en effet, ne pouvant plus conduire il ne se déplace qu'en transports en commun. Il partage son temps libre entre la pêche, les courses de voiliers miniatures sur les étangs d'Apigné (+ reliance : loisirs), et des recherches historiques sur son village natal qu'il effectue au centre de documentation des Champs Libres (+ reliance : culture) une fois par semaine.

Très attaché au monde rural il lui semble vivre un paradoxe étrange : homme de la campagne, il habite désormais un quartier qu'il a vu se construire au fil 
des ans et ne reconnait rien de ce qu'il a connu auparavant (- déliance : rupture avec le passe). La richesse du patrimoine rural qui parsème le territoire le rend parfois nostalgique, lors des promenades dominicales qu'il fait avec ses enfants.

M. n'est pas attaché à la ville, mais y vit par commodité, il trouve cependant à la Morinais un juste compromis entre ses aspiration d'indépendances et la qualité de vie (+ reliance : équilibre).

\subsubsection{L'eau, la terre, le ciel, pour tous ? / L., l'éclusier}

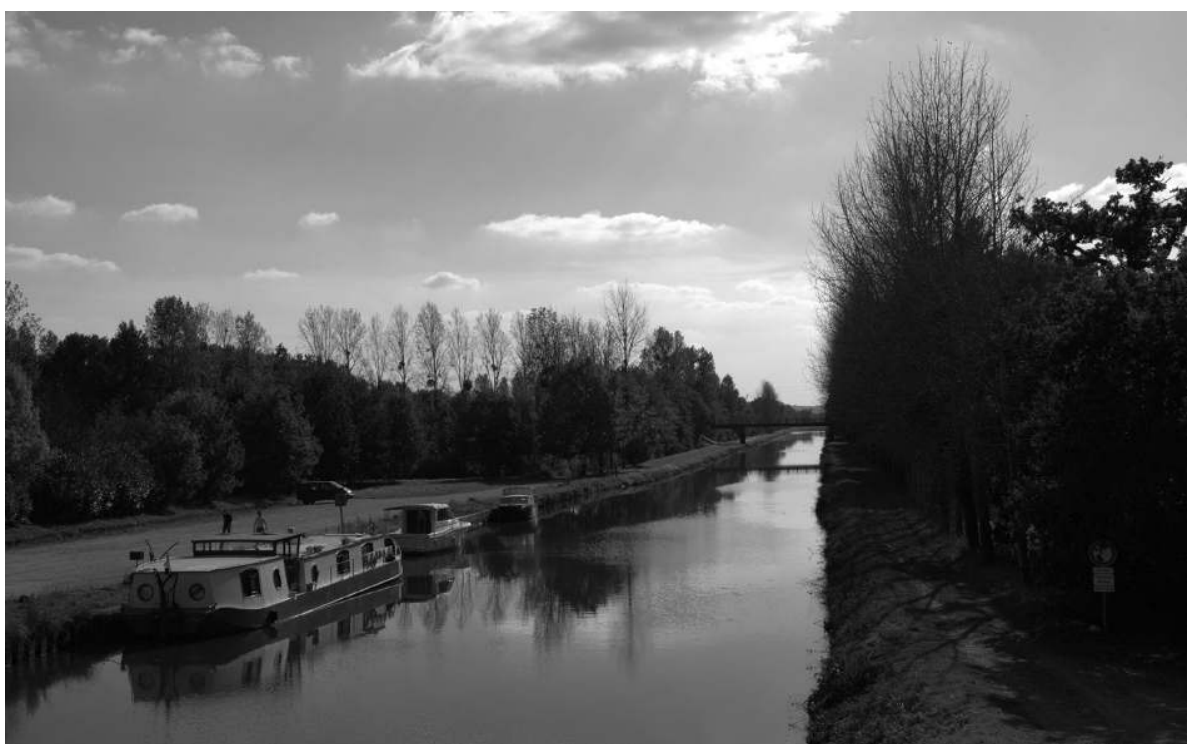

Illustration 5. La Vilaine canalisée (source : MAD).

L. est éclusier et adore son métier. Avant d'être posté sur l'écluse du canal de la Vilaine il travaillait sur le canal de Nantes à Brest et l'activité y était autrement plus stimulante (- déliance : perte de représentation sociale)! En effet L. compte ici le passage des péniches sur les doigts de la main. Alors pour passer le temps il bricole et discute avec les passants : plaisanciers, promeneurs, joggers ou pellerins [le canal est en effet sur le chemin de St-Jacques de Compostel (+ reliance: accès au lointain)]. Conscient de la beauté du lieu qu'il habite il s'improvise parfois poète (++ reliance : poétique des éléments naturels) et médite cette ligne de ciel parfaitement droite, observe les effets du vent sur sa surface, et ce pendant de longues minutes...

L. regrette que le canal ne soit pas plus exploité, il trouve dommage de ne pas utiliser une infrastructure déjà en place, en parfait état de marche. Il sait par cœur les distances au prochain camping, point de ravitaillement ou restaurant et répond invariablement "non vous ne trouverez pas cela ici " aux questions des gens qu'il rencontre. Les activités sont trop éloignées de la 
rivière (-- déliance : absence de connections), et depuis que l'exploitation des carrières se fait par voie routière le canal est sous utilisé.

Depuis qu'il est arrivé ici il constate cependant que de plus en plus de marcheurs ou de cyclistes viennent de Rennes (+ reliance: accessibilité Rennes) jusqu’à lui. Il trouve cela étrange d'ailleurs qu'il soit plus facile de longer la Vilaine pour un Rennais que pour les habitants du coin (- déliance : éloignement du proche). Mais pas plus de plaisanciers... Même lorsqu'il est posté dans sa baraque on lui demande régulièrement si la rivière est navigable (-déliance : méconnaissance)!

\subsubsection{La vie étudiante / C., étudiante à Ker Lann}

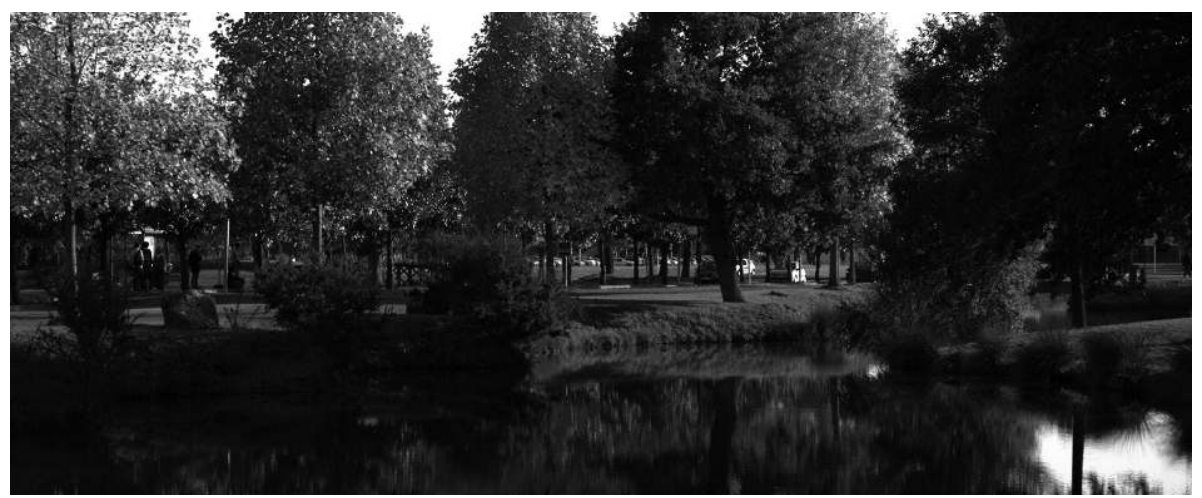

Illustration 6. Le campus de Ker Lann (source : MAD).

C. a 21 ans, elle est étudiante à l'ENSAI (École de statistique et de traitement de l'information) sur le campus de Ker Lann mais n'ayant pas de voiture et faute d'avoir trouvé un logement plus près (- déliance : éloignement forcé), (il n'est pas facile d'obtenir un logement dans les résidences du campus) elle habite le centre de Rennes. Originaire du Mans il est vrai qu'elle souhaitait n'être pas trop éloigné de la gare car elle retourne souvent voir sa famille $(+$ reliance: proximité familiale). Et puis la vie étudiante, c'est aussi les sorties entre amis, la découverte de l'indépendance et les premiers jobs (elle est serveuse deux soirs par semaine dans un bar de son quartier). Pour toutes ces raisons la vie en ville l'attirait davantage, et habiter le centre de Rennes lui convient tout à fait.

Le matin elle met environ trente minutes pour se rendre de chez elle à l'école (+ reliance : accessibilité centre / périphérie). Les emplois du temps sont compacts, elle n'a que rarement une heure de libre entre deux cours, ce n'est pas comme à la fac, et heureusement pense-t-elle car elle ne saurait trop quoi faire (-déliance : ennui) sur place pour s'occuper. Chaque midi elle déjeune dans un des restaurants universitaires du campus, puis l'après-midi s'enchaîne : elle suit ses cours jusqu'à reprendre le bus en fin d'après-midi.

Ayant toujours vécu à la campagne la présence du végétal et de l'eau lui plaise (+ reliance : proximité nature) et dans l'ensemble elle trouve l'environnement 
du campus très agréable et propice au travail. Un peu trop calme à son goût c'est vrai, aussi le soir apprécie-t-elle l'agitation de la rue St-Michel et la proximité des bars et restaurants du quartier.

2.2.5. Une place dans la société / B., ouvrier chez PSA (contexte 2010)
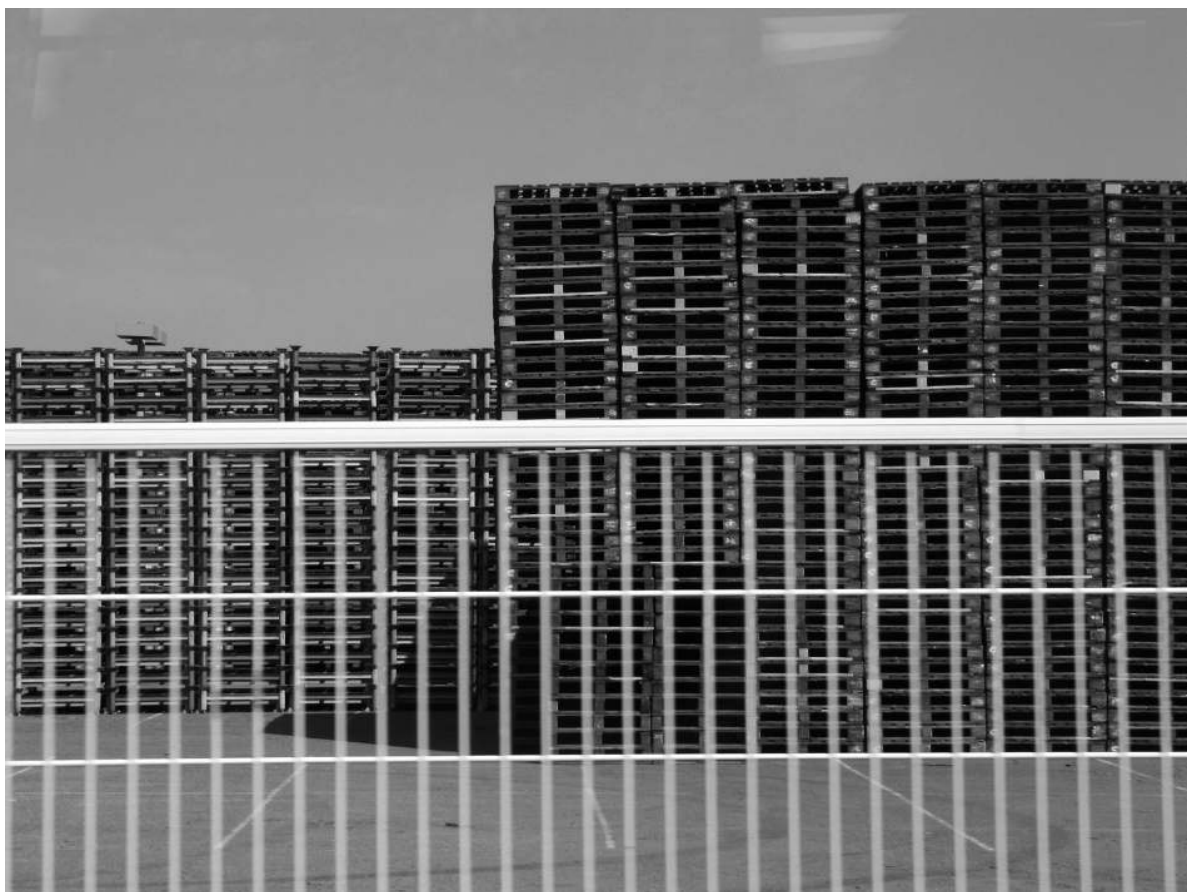

Illustration 7. Le site PSA en 2010, stockage (source : MAD).

B. a 20 ans, il habite chez ses parents à Chavagne et travaille chez PSA depuis 2 ans. Tous les jours il traverse la Vilaine et passe "de l'autre côté ». Il n'utilise pas le mot " rive " parce que ce qu'il traverse n'est pas à proprement parler une rivière mais une vallée. Il connait par cœur les routes qu'il empreinte : ce sont les mêmes qu'il empruntait avec sa mère quand elle le déposait au Lycée Brequigny, au Sud de Rennes, avant de se rendre au travail dans le centre. À présent il a sa propre voiture et se rend chaque matin avec sur le site de la Janais. Il aime ces petites routes et l'impression de traverser une épaisseur végétale (++ reliance : sentiment de vivre au milieu de la nature). Il aime vivre à la campagne même s'il préfere, le week-end, retrouver ses amis à Rennes. Il ne voit cependant pas l'intérêt d'aller vivre à Rennes, car c'est "à peine à dix minutes d'ici » (+ reliance : proximité de Rennes).

Il aime son travail car il participe à la construction de voitures haut de gamme. Le fait d'intégrer un groupe tel que PSA a éveillé sa conscience sociale 
(++ reliance : représentation sociale). Il est parmi des milliers d'autres ici à Rennes à faire les trois huit, et parmi des dizaines de milliers d'autres ailleurs en France et en Europe, tous un peu comme lui. Beaucoup de ses amis aujourd'hui sont d'ailleurs des collègues de travail et la solidarité qui s'est installée autour de lui depuis son arrivée le rassure un peu en ces temps de crise (- déliance : incertitude de l'avenir). Il se dit « on est tous dans le même bateau... » et c'est important pour lui.

Bien que célibataire ses aspirations ne vont pas encore vers la construction d'une famille. Il se trouve trop jeune pour ça et rêve plutôt de voyages (+ reliance : ailleurs / voyage). Souvent le matin, quand il passe devant l'aéroport, il se dit qu'il aimerait connaitre l'Australie mais il ne sait pas qu'aucun avion ici ne s'envole pour Sydney.

\subsubsection{Un monde nouveau a recouvert l'ancien / H. habite le quartier de St-Jacques Aéroport}

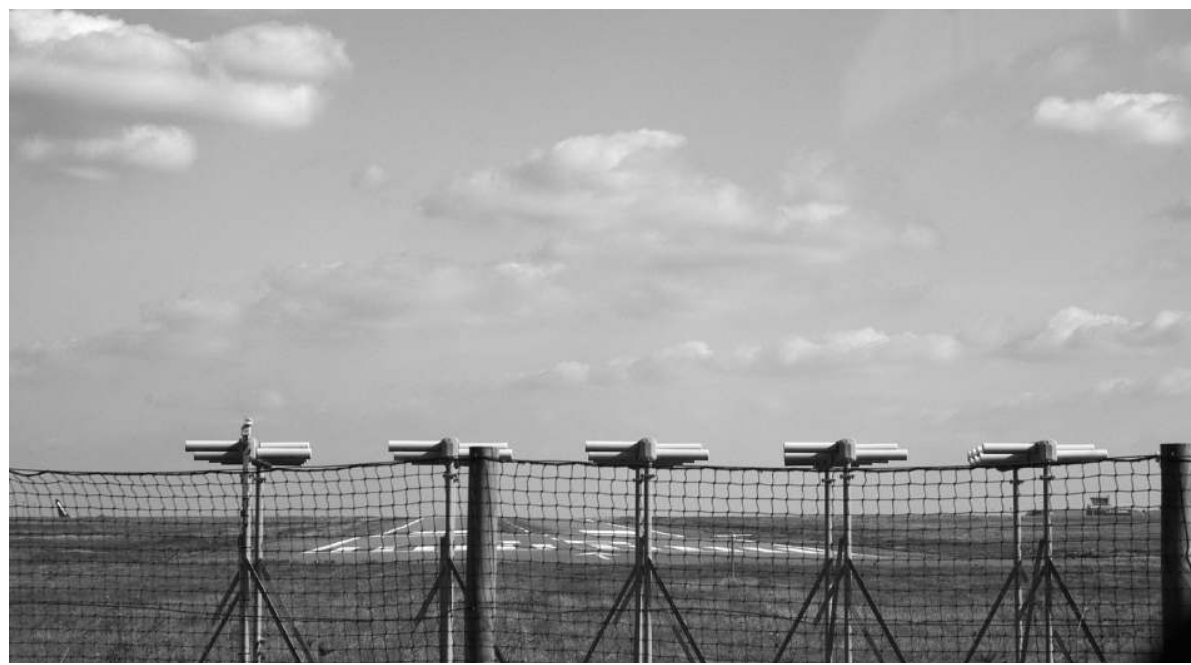

Illustration 8. L'aéroport Rennes-St-Jaques (source : MAD).

H. a 80 ans et vit à Saint-Jacques-de-la-Lande depuis toujours. Elle se souvient du temps où le centre c'était ici. Elle se souvient des commerces, de la vie animée du petit bourg qu'elle n'a jamais quitté. Elle se souvient de la guerre, des destructions et de l'extension de l'Aéroport qui borde presque aujourd'hui sa maison. Elle se souvient aussi de la construction des usines Citroën. Elle a vu peu à peu les zones d'activités remplacer les champs de son enfance (- déliance : rupture avec le passé). Elle a vu les petits commerces déserter sa ville. Elle a vu l'essor de l'automobile et aujourd'hui le trafic, et tous ces camions chaque jour. Elle a vu récemment se construire une ville qui porte le même nom que la sienne : Saint-Jacques-de-la-Lande, à quelques kilomètres de là, mais qui n'est pas la sienne (- déliance : absence de sentiment d'appartenance). 
Elle a vu s'allonger les distances, elle qui allait à pied jusqu'à la Vilaine et se promener dans la campagne pendant des heures, et qu'aujourd'hui tout lui parait trop loin (- déliance : inaccessibilite). Aujourd'hui elle regarde la campagne au loin (+ reliance: horizon), elle dit qu'elle ne fait plus attention à l'aéroport, que son regard passe au-dessus. Elle a vu des milliers d'avions décoller mais n'a jamais voyager. Elle a vu des milliers d'avions décoller si bien qu'elle ne les entend plus (- déliance : inconfort sonore). Elle vit comme dans une bulle dont elle ne sort pas, coincée entre l'aéroport, le parc expo, et les usines PSA (-- déliance: isolement). Elle vit dans un monde qu'elle ne reconnait pas, un monde nouveau qui aurait recouvert le sien.

2.2.7. Une journée type / la famille Z., centre sud de Bruz

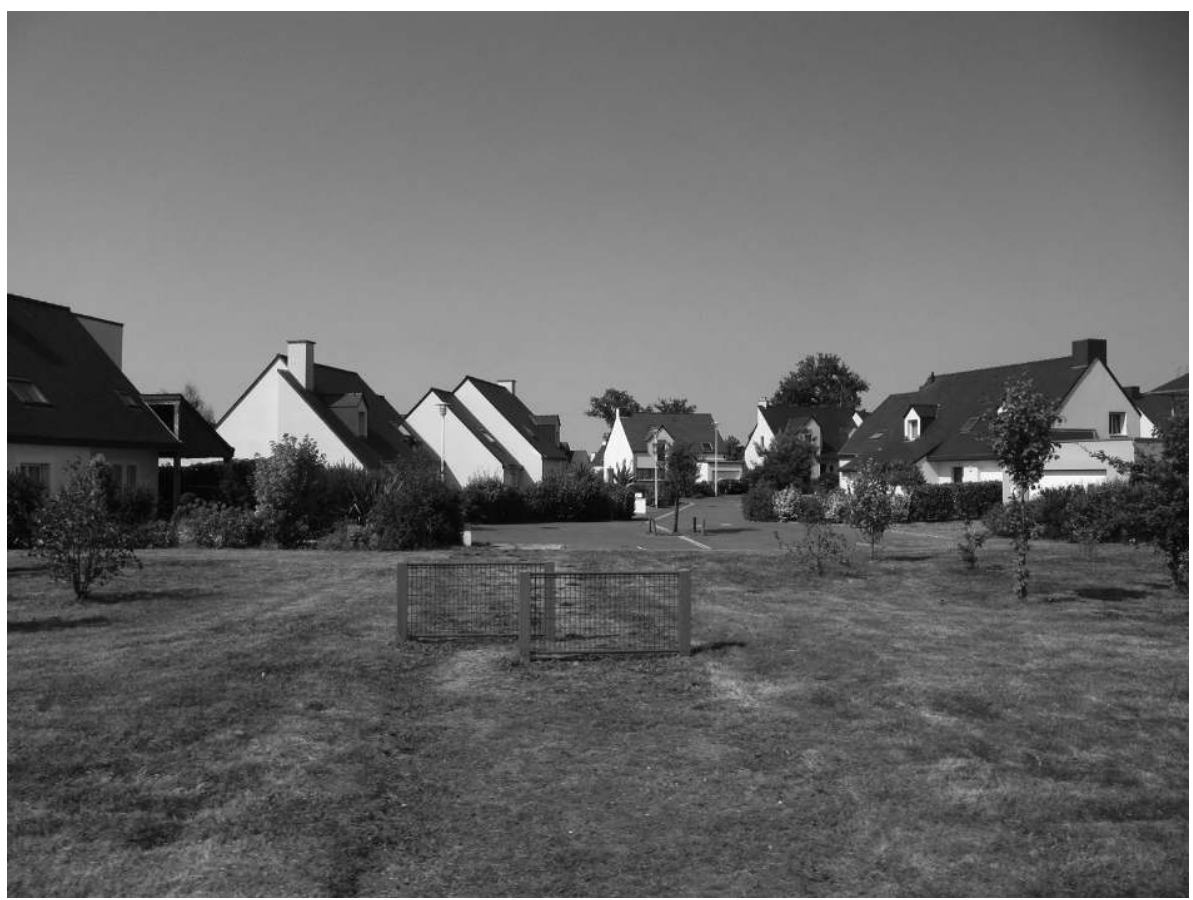

Illustration 9. Un lotissement pavillonnaire à Bruz (source : MAD). 
Le matin Madame Z. quitte sa maison $7 \mathrm{~h} 30$ et roule jusqu'à la gare de Bruz pour prendre le train de $7 \mathrm{~h} 39$, qui l'amènera à Rennes en 11 minutes (+ reliance : accessibilité centre / périphérie), il ne lui restera plus qu'à parcourir environ 500 mètres pour arriver sur son lieu de travail. Elle se dit qu'elle ne met finalement pas plus de temps qu'il y a quatre ans, alors qu'avec son mari ils habitaient encore à Rennes. Le soir elle prend le train de $17 \mathrm{~h} 16$ et va chercher sa fille à la garderie de l'école Jacques Prévert, s'arrête souvent dans le centre de Bruz pour y faire quelques courses sans jamais s'étonner du fait que ce " centre " ne soit qu'un vaste parking (-- déliance : surutilisation de la voiture). À $18 \mathrm{~h}$ elle est chez elle.

Monsieur Z. part un peu plus tard le matin. Il dépose sa fille peu avant $8 \mathrm{~h}$ à l'école puis file au travail, dans la ZAC de la Touche Tizon où il est cadre chez un important prestataire de PSA. Venir habiter à Bruz lui a fait gagner beaucoup de temps (+ reliance : vie de famille), il peut même déjeuner chez lui chaque midi, avec son fils, entré cette année au collège Pierre Brossolette situé à quelques centaines de mètres de la maison (+ reliance: moins de déplacement). À $19 \mathrm{~h}$ il est chez lui.

Le soir ils se retrouvent facilement entre voisins pour un apéritif. Ils se rendent beaucoup de services (+ reliance : bon voisinage) : pour la garde des enfants, un dépannage alimentaire... Ce sont d'ailleurs les enfants, qui sont à peu près tous de la même génération, qui ont souvent été à l'origine des relations entre les habitants du lotissement. Ils sont mieux ici qu'à Rennes pense madame Z.

Plus tard ils envisagent le week-end approchant : une pièce de théâtre au Grand Logis (+ reliance : accessibilité culture) samedi soir ? Un déjeuner dimanche ? Et pourquoi pas inviter les $\mathrm{R}$ ? Ils se disent que s'il fait beau ils iront faire un tour l'après-midi ensemble au moulin du Boël (+ reliance : proximité nature, patrimoine). 
2.2.8. La colère du golfeur / V. habitant du golf de Cicé-Blossac et son ami G.

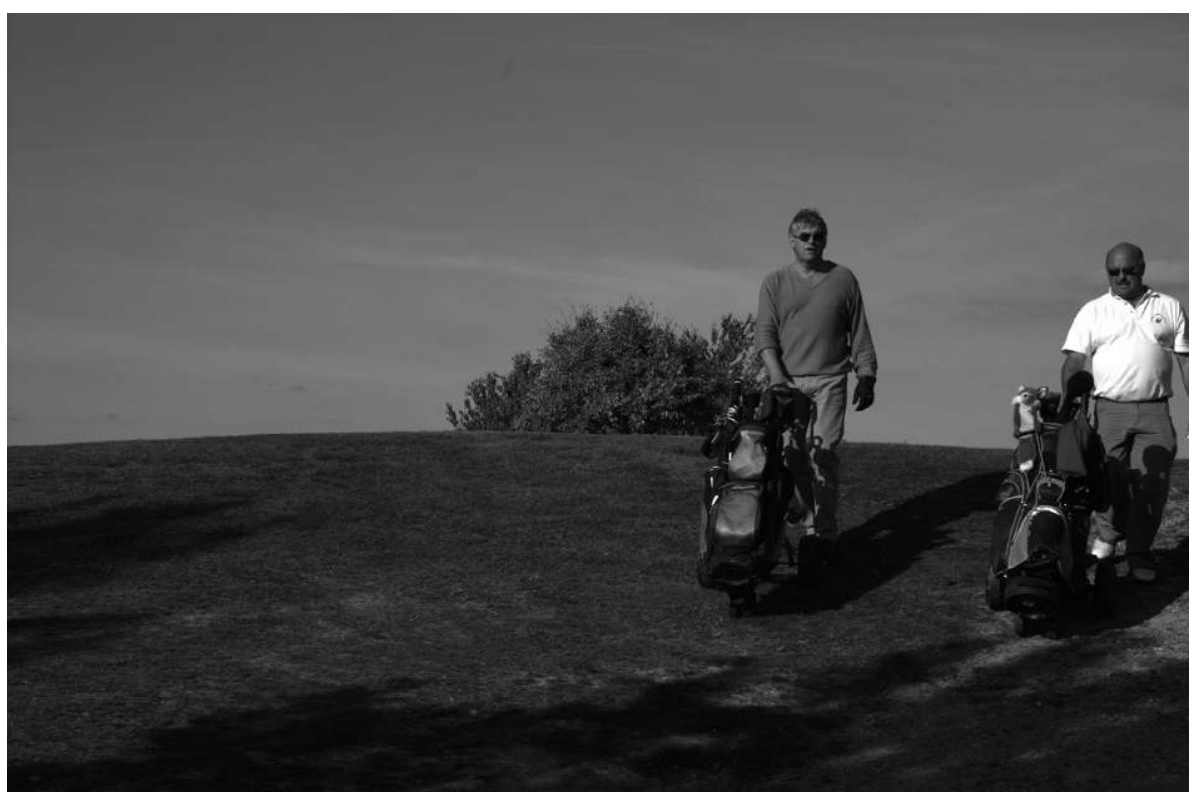

Illustration 10. Golfeurs au golf de Cicé-Blossac (source : MAD).

Vivant à St-Malo G. est chef d'une petite entreprise de transport public. Pour des besoins professionnels il fait régulièrement des déplacements à Rennes, afin d'y rencontrer de nouveaux clients et partenaires. Parfois il rend visite à son ami et ancien collègue $\mathrm{V}$, , qui vit dans une de ces maisons du golf de Cicé-Blossac, et chaque fois G. s'émerveille de la proximité de la nature et de la ville (+ reliance : proximité de la nature).

G. et V. étant tous deux golfeurs, chaque rencontre est l'occasion d'une partie aussi amicale que disputée. Ce jour-là cependant G. est déçu du parcours. Comparativement avec les autres golfs de la région il avait toujours trouvé celui-ci relativement laid. Bien que le nombre de pièces d'eau le rendait techniquement intéressant (+ reliance: rapport aux éléments), la proximité des maisons et des routes lui donnaient l'impression de jouer dans un lotissement. Le rapport au paysage était gâché (- déliance : rapport déprécié à la nature). Et la quatre voies était trop bruyante. Mais là c'est l'entretien qui l'exaspérait : fairway mal tondu, pregreen chaotique, herbe dans les bunker, foin dans les roughs, greens autrefois rapides et dures plus lents et mous. Il fit part de son agacement à V., qui s'agaça à son tour et le ton monta d'un cran, G. allant jusqu'à dire que si ce golf était prisé dans la région ce n'était que parce qu’il était surévalué et qu'il permettait de baisser facilement son index. La discussion s'envenima rapidement, et dérivant du tout au tout $\mathrm{G}$. se mit à critiquer le mode de vie 
« entre-soi » (--- déliance : ségrégation sociale) de V., cette façon de s'enclore, ce petit lotissement presque "à l'américaine ", il alla même jusqu’à moquer l'entrée en fausses pierres, ridicule selon lui.

C'est la dernière fois que G. mit les pieds au golf de Cicé-Blossac.

\section{Transition}

Dans ce premier cas, récits de vie et projet de territoire convergent vers une même aspiration à éco-habiter la métropole rennaise et travaillent un vivre-ensemble, pacifié, mesuré et équilibré. L'adéquation entre les propositions des auteurs du projet et les attentes des destinataires n'en est que plus forte. L'usage du récit assume et facilite cette médiation, les vertus liantes (ou reliantes) du récit ont contribué à la constitution d'un récit collectif partageable - " habiter le bocage métropolitain » : à partir de la diversité des situations habitantes rencontrées, le projet a pu ouvrir une direction commune.

Mais ce n'est pas toujours le cas, le commun ne tient pas nécessairement dans la reconnaissance unanime d'une aspiration à suivre, il peut aussi se révéler dans la peur. À Bruxelles, quartier Masui, 26 communautés cohabitent et pour toutes, raconter, se raconter, c'est d'abord la nécessité de dire la provenance avant de pouvoir ne serait-ce qu'évoquer la moindre projection. Quartier d'accueil pour de nombreux immigrés, où règne le caché, le secret et le système D ; ce que l'on recueille : des histoires au futur conditionné, voire absent. Le récit devient alors l'outil et l'expression muette du multiple, du résistant et du solidaire. Là où toute irruption institutionnelle est perçue comme antigène, le récit sert le projet en sa capacité d'écoute et œuvre à la reconnaissance et au ménagement du milieu en présence.

\section{Récits de vie et mémoires / Cas $n^{\circ} 2$ : Bruxelles, Masui}

Depuis 2011, nous travaillons en étroite collaboration avec l'agence de paysagiste parisienne La Compagnie du Paysage (Jean-Frédéric Gay et Nathalie Melin) au projet d'aménagement du parc de la Senne (mené par l'Institut Bruxellois pour la Gestion de l'Environnement), dans le quartier Masui (partie ancienne du quartier Nord de Bruxelles). Ce quartier tout entier vit sous la pression d'un gigantesque projet urbain depuis plus de quarante ans. Nommé pompeusement Manhattan dans les années 1960, le spectre de cette ambition quasi science-fictionnelle rôde encore dans les rues au point de priver les habitants de Masui de parole dès qu'il s'agit de mettre en projet leur quartier. Pire, leur mutisme paraît même désincarner tant le futur du quartier semble distinct du leur. Le projet prend place sur l'ancien lit de la Senne, rivière détournée puis canalisée dans les années 1950.

Travaillant avec le réseau social en place, nous avons intitulé une première partie de cette étude : "Faire milieu avec l'invisible ». En voici une synthèse. 


\section{Le fantôme de Manhattan}

"Davantage qu'une ville, le Manhattan sera un monde...»

"Vivre au Manhattan Center sera, désormais, l'expression la plus pleine du mot "vivre"..."

"Le monde entier passera au Manhattan Center..."

Les promoteurs du Manhattan Center. Source : www.quartiernord.be ${ }^{13}$

"Le projet Manhattan fut élaboré dans les années 1960. Il s’agissait de raser purement et simplement, au nom de l'utilité publique et en extrême urgence, 53 hectares de tissu urbain pour reproduire au niveau de la capitale belge un quartier d'affaires semblable à celui de New York, relié au reste du continent par héliport et autoroutes. Conçu par le groupe Structures, approuvé par les arrêtés royaux du 17 février 1967, sa réalisation va provoquer, dans sa première phase c'est-à-dire "la libération du sol", parmi les habitants expropriés et/ou expulsés du quartier Nord, des drames humains à répétition pour accoucher péniblement, quatre décennies plus tard, de ce qu'un journaliste a appelé un "quartier bâtard". "

Les habitants du quartier Nord. Source : www.quartiernord.be

Même s'il ne ressemble en rien au projet de départ, aujourd'hui encore Manhattan résonne entre les tours de ce " quartier bâtard ». À chaque nouveau projet, à chaque nouveau chantier "C'est encore Manhattan qui gagne du terrain ». Invoquée de manière explicite par les anciens, ou dissimulé sous le pronom personnel - « On sait qu'ILS ne font pas ça pour nous, c'est pour en attirer d'autres ", "ON a commencé à démolir en 1972 " $^{14}$ - la même menace guette, silencieuse, invisible, mais prégnante. Au point de questionner : de quoi Manhattan est-il le nom ? Ou mieux encore : de quoi est-il le non-dit ?

Manhattan, c'est d'abord un vieux projet hanté, comme un cauchemar qu'on ne pourrait oublier. Pour les plus anciens du quartier, ceux qui ne sont restés que parce qu'ils se trouvaient du bon côté de l'avenue de l'héliport en 1967, Manhattan est un souvenir douloureux : la perte de quelques amis chers, expulsés on ne sait où, la démolition des rues et des immeubles en briques, et avec, une ambiance chaleureuse et populaire, la négation du passé ouvrier et de la culture bigarrée qui sont les leurs, la disparition des souvenirs d'enfance.

Manhattan, dit ou non-dit, représente donc pour les habitants de Masui l'histoire violentée du quartier Nord, et réciproquement, Masui représente les vestiges d'un temps révolu, âge d'or du quartier Nord (d'ailleurs on l'appelle l'« ancien» quartier).

13 Le site Internet www.quartiernord.be est un site référent regroupant une somme d'informations et de témoignage d'habitants ayant vécu la mutation du quartier. La vivacité passée des associations de quartiers marque d'ailleurs un terrible contraste avec la situation actuelle.

14 Les citations de ce paragraphe sont tirées des comptes-rendus de la consultation menée par l'agence bruxelloise d'architecture et d'urbanisme Karbon dans le cadre de sa mission de coordination du Contrat de Quartier Durable Masui menée en 2010. 
Pour les habitants du quartier Masui, Manhattan représente aussi l'inexorable de leur futur, une fatalité, comme une marche du monde contre laquelle ils ne peuvent rien. Les travaux engagés dans les années 1960 ne se sont jamais arrêtés jusqu'à aujourd'hui, quelles raisons aurait-on de ne pas les continuer ? La mutation du quartier Nord a connu une phase qui a entièrement modifié son sol, effacé des rues, rayé de la carte des îlots entiers ; tout le monde s'attend à ce qu'ils connaissent une phase 2 , plus souterraine peut-être, mais néanmoins toute aussi dévastatrice.

Manhattan, c'est finalement le nom de la peur. La peur d'être « repoussés jusqu'au canal ", et même pas au-delà, " jusqu'au ", comme pour dire qu'un jour ou l'autre la gentrification les poussera dedans. Alors bien sûr, aucun intérêt à accélérer les choses, les habitants se cachent, se taisent, se font discrets, attendent. Le quartier Nord c'est une histoire collective en marche, sourde, et qui les dépasse, qui ne laisse aucune place aux récits singuliers, qui ne les entend pas. Et affaiblie, évidemment, la communauté en fragments, incapable de parler d'une seule voix, et encore moins d'une seule langue. Alors, à l'ère des discours identitaires unifiant, englobant, embrassant, comment faire quand on est sans voix ? L'éclaté, le multiple, le singulier est toujours compliqué, insaisissable et pour finir invisible.

\section{La communauté invisible}

Il n'y a pas de vue d'ensemble, il faut l'accepter. "Les habitants " n'existent pas sous une forme unique et arrêtée, leur communauté est invisible, leurs revendications et leurs aspirations à la limite de l'indicible, il faudra faire autrement. Pour comprendre le multiple, Deleuze et Guatari insistaient sur l'importance de la relation ou de l'interrelation entre plusieurs éléments, l'importance de la qualification du passage de l'un à l'autre, l'importance au fond, de ce qui les unit, de leur solidarité. Alors, ce milieu habité qu'est le quartier Masui, qu'est-ce qui fait que, même fragile, "ça " tient ensemble ? Notre hypothèse est de dire que ce sont la mémoire, et les mémoires, qui fondent l'identité du quartier en tant que milieu. Et que la projection dans le futur qui est proposée par le projet de ce parc sur lequel nous travaillons, ne sera acceptée (entendue) par les habitants qu’à la condition qu'il préserve ce milieu.

Les murs du quartier Masui sont faits de briques et de souvenirs. Cette capacité, du matériau brique, à se laisser imprégner par le temps n'est pas celle des tours de verre du nouveau quartier. (Comme le soulignait déjà Walter Benjamin, ${ }^{15}$ les architecture de verre sont lisses, elles ne marquent pas la trace du temps et au final sont anhistoriques.) Que l'espace contiendrait,

15 Voir sur le sujet Walter Benjamin, Paris : capitale du XIXe siècle : le livre des passages, Éditions du Cerf, 1997 (3 réed.), 972 p. 
en sa matérialité même, quelque chose de l'ordre du temporel, l'idée n'est pas nouvelle, mais son rappel s'inscrit dans une pensée qui nous paraît particulièrement à propos dès lors qu'il s'agit d'intervenir dans un quartier tel que le quartier Masui : l'architecture et le paysage sont aussi, et peut-être surtout, des arts de la mémoire.

Cette question de la mémoire, et donc de la considération du temps, dans la pensée du spatial occupe une place centrale dans la réflexion théorique contemporaine sur l'architecture et le paysage : des philosophes comme Sébastien Marot ${ }^{16}$ ou Gilles Tiberghien ${ }^{17}$ en sont aujourd'hui les fers de lance, la redécouverte des travaux de Frances Yates ${ }^{18}$, ou de Maurice Hallwachs ${ }^{19}$ y sont pour beaucoup. (La première exposition sur la Tendenzia au centre Pompidou à Paris qui s'est tenue cet été n'en est que la confirmation, Yates et Hallwachs étant des références privilégiées dans les travaux d'Aldo Rossi.) Comme le reprend Marot, ce dernier affirmait « (...) l'espace est une réalité qui dure : nos impressions se chassent l'une l'autre, rien ne demeure dans notre esprit, et l'on ne comprendrait pas que nous puissions ressaisir le passé s'il ne se conservait pas en effet dans le milieu matériel qui nous entoure. (...) $»^{20}$ Ainsi, l'architecture et le paysage concrétisent le temps, et visent des effets de présence. Ils matérialisent l'épaisseur du présent, ils le triplent comme l'écrivait Saint-Augustin par un présent du passé (le souvenir), puis par un présent du futur (l'attente) faisant spécificité de l'édification de cet état de présence qui ouvre à l'expérience poétique, expérience du récit en tant qu'irruption et prolongement de l'Histoire dans et par l'histoire personnelle de chacun. L'espace commun et partagé est ainsi celui-là même où cohabitent, se croisent et interagissent mémoire collective et subjective, souvenirs anthologiques et anecdotiques, histoire du lieu et des trajectoires des vies de chacun.

Cette place ou ce ménagement des récits de vie par le projet nous paraît d'autant plus essentiel que ces récits de vie sont difficiles à saisir, presque inaudibles. L'erreur serait de penser le projet comme unifiant, ou fédérateur. L'attention à porter à l'identité des habitants du quartier ne peut se faire qu'en laissant place au multiple. Pour le philosophe Jean-Luc Nancy l'identité est toujours plurielle : "simple index tendu [...] dans la direction de cela qui vient, qui ne cesse de venir, qui revient et qui se transforme, qui fraye des voies nouvelles, qui laisse des traces, mais jamais une chose ni une unité de sens ",

16 Sébastien Marot, L'art de la mémoire, l'architecture et le territoire, Éditions de la Villette, 2010, $142 \mathrm{p}$.

17 Giles Tiberghien, Notes sur la nature, la cabane et quelques autres choses, Édition du Félin, 2005, $152 \mathrm{p}$.

18 Voir : Frances Yates, L’art de la mémoire, Éditions Gallimard, 1987 (éd. Française), 432 p.

19 Voir : Maurice Halbwachs, Les cadres sociaux de la mémoire, Albin Michel, 1994 (éd. Française), $374 \mathrm{p}$.

20 Sébastien Marot, ouvrage cité. 
avant de prévenir : «Sans travail, sans lieux ni conditions de vie autres que les sous-produits d'urbanisation sans urbanité, sans formations conçues autrement que par rafistolages de modèles périmés, il est impossible de seulement envisager un horizon d'“identités", même quand on ne désire rien d'autre. Et il est alors naturel qu'on se replie dans de petites identités séparées, suridentifiées par leur séparation, durcies, exaspérées. $»^{21}$

Vingt-six communautés, les unes à côté des autres, cohabitent dans le quartier Masui. Malgré les tensions et les difficultés qui parfois apparaissent, elles ont trouvé une forme d'urbanité discrète, une distance polie entre elles qu'il s'agit de respecter. Cet espace intercommunautaire que le projet qualifiera et aménagera, c'est cela la volonté habitante : la préservation de leur milieu.

\section{Le parc n'est pas un parc, la rivière n'est plus une rivière}

Lors de la visite organisée de site nous avons vu a quel point le fait que le parc, à l'heure actuelle, était invisible et cultivait une conception de l'espace public comme jardin secret. L'émerveillement des quelques habitants découvrant l'arrière de l'école de l'Allée Verte est de ce point de vue significatif : les habitants adhèrent à l'idée selon laquelle leur quartier a quelque chose de mystérieux et est détenteur de quelques trésors cachés. C'est sans doute sous cette forme que le parc existe en premier lieu : sous la forme d'un chapelet de jardins secrets, cachés en coeurs d'îlots. Le projet appelle ainsi un rapport à l'intimité, à l'entre soi, au secret partagé. Quand le parc sera fermé il deviendra jardin, quatre fois jardin. Cette fermeture et donc fragmentation du projet la nuit venue n'est pas qu'une question de sécurité, c'est aussi une question de représentation : les deux temporalités du parc induisent deux états d'existence. L'inquiétude immense que suscite le contrat de quartier et le projet Parc de Senne auprès des habitants et la prégnance, la résistance du milieu, nous permettent d'affiner l'image que nous fabriquons pour ce "parc de Senne » en même temps que de questionner son nom. En effet cette liaison très fine traversant et reliant les différents points du quartier Masui, n'a rien de ce que l'on nomme communément par la dénomination " parc ». C'est une allée, une promenade, un corridor, une sente ; le jour c'est une sente. L'idée de parc induit la représentation d'usages majoritairement statiques (Ces usages statiques, sont déjà-là, latents. Ce sont toutes ces demandes et attentes qu'ont exprimées les personnes $\mathrm{d} u$ groupe relais) alors que l'aménagement créé sera fait d'abord pour le déplacement.

Jardins la nuit, sente le jour, le Parc de Senne n'est pas un parc ; la Senne non plus d'ailleurs, n'est plus une rivière. On comprend alors l'intérêt d'une réflexion multiscalaire. Le parc à l'échelle de l'îlot devient jardin (précision que le terme de jardin, s'il est plus domestique et intime que celui de parc peut tout à fait

21 Jean-Luc Nancy, Identité : fragments, franchises, Éd. Galilée, 2010, 69 p. 
renvoyer à un lieu public : exemple, les jardins d'Éole, à Paris). À l'échelle du quartier il devient une liaison, un cheminement, une sente avons nous dit. Enfin, à une échelle communale ce parc se confond avec le tracé de la rivière disparue. Chaque échelle de représentation dévoile un degré de visibilité du projet.

Amas de micro-mondes pris dans les flux du monde, le quartier Masui cherche en même temps à se protéger et à se connecter, cherche à ménager et l'intime et le commun. Penser le parce de Senne en tant que «sente métropolitaine " c'est penser cette articulation du micro et du macro, en gardant à l'esprit que ce l'on cherche au contact de la nature n'est rien d'autre que l'apaisement.

En tant que vestige qui a une valeur propre de témoignage de résistance du lieu, le quartier Masui donne consistance au déracinement des populations qui l'habitent. Réceptacle ouvert aux mémoires de chacun, il s'adapte en permanence, il se métamorphose. Son histoire est celle du constant renouvellement de sa capacité d'accueil des nouveaux habitants. 


\section{Conclusion}

Construire et aménager pour les autres : la notion de milieux en appelle au partage. Partage des expériences, partage de l'espace, partage des cultures, partage des mémoires, partage des projets. Mettre en partage ce qui nous relie les uns aux autres. Se mettre à la place de l'autre.

L'empathie est une qualité difficile à cultiver mais néanmoins indispensable aux architectes et aux urbanistes. Concepteurs, ils n'en demeurent bien sûr pas moins usagers des espaces qu'ils conçoivent, habitants des immeubles qu'ils construisent ; mais cette condition duale, cette expérience entre, est fragile. Le récit permet d'habiter cette transmission, et à ce titre, est un outil intéressant qui replace le destinataire au centre des préoccupations de représentation et de conception des milieux mis en transformation.

Chez Ricœur, le récit préfigure, configure et refigure. Il est chaque fois l'œuvre conjointe d'un déjà-là, d'un auteur et d'un lecteur. Un jeu d'équilibre s'instaure alors et l'intertextualité que le philosophe décrit comme les interactions effectives entre texte écrit et texte lu appellerait à décrire, de manière analogue, la qualité d'" interurbanité " des milieux habités. C'est cela même qui constitue l'objet de cet article, qui vise, par l'étude de deux cas, à ouvrir le champ des représentations des milieux habités à partir d'une pensée de la mise en partage et de la transmission des projets architecturaux et urbains, car pour le dire avec Edgar Morin, nous sommes toujours engagés dans la recherche à l'œuvre d'une " collaboration du monde extérieur et de notre esprit pour construire la réalité $»{ }^{22}$

Marc-Antoine Durand Architecte DPLG École Nationale Supérieure d'Architecture de Paris-la-Villette Laboratoire GERPHAU - UMR 7218 MCC/CNRS - LAVUE info@mad-architecture.com

22 Daniel Bougnoux et Bastien Engelbach, «Entretien avec Edgar Morin (2) : Science et philosophie", www.nonfiction.fr, 10 avril 2008. 